\title{
Valoración de un sistema de puntuación de parámetros múltiples de la prueba sin estrés
}

\author{
Iván Jaramillo Arias*; Jenny Muñoz Rengifo**; Braulio Lara Alvarez***
}

\begin{abstract}
RESUMEN: Aunque el Test de no estrés, es un método ampliamente aceptado de vigilancia fetal, uno de sus problemas ha sido la falta de estándares para su interpretación. Se valoraron prospectivamente 142 pruebas, realizadas en 90 pacientes, utilizando un sistema de calificación de parámetros múltiples que cuantifica cinco variables: frecuencia cardíaca basal, frecuencia y amplitud de las oscilaciones, aceleraciones y desaceleraciones. Empleando estrictos parámetros de confirmación del Bienestar Fetal, el sistema tuvo una sensibilidad del $50 \%$, especificidad del $\mathbf{9 8 . 4 \%}$ y exactitud del $96.9 \%$. El análisis de las mismas pruebas utilizando solamente criterios de reactividad dio una sensibilidad del $75 \%$, especificidad del $92 \%$ y exactitud del $91.6 \%$.
\end{abstract}

La cuantificación de parámetros múltiples del TNS permiten una lectura fácil y objetiva de los trazados, independiente de la presencia de movimientos fetales. Facilita el análisis de trazados de dudosa reactividad y unifica criterios de interpretación para comparar estudios entre grupos e instituciones.

PALABRAS CLAVES: TNS. Sistemas de interpretación.

SUMMARY: Although NST is a widely accepted method of fetal monitoring one of its problems has been the lack of standars for its interpretation. One hundred forty two (142) NSTs were evaluated prospectively on 90 patients, using a system of evaluations of multiple parameters in which five variables were measured: Baseline rate, frecuency and breadht of the fluctuations, accelerations and decelerations.

Employing strict parameters of confirmation of the fetal well-being, the system had a sensibility of $50 \%$ a specificity of $98.4 \%$ and an accuracy of $96.9 \%$. The analysis of the same tests utilizing only criteria of reactivity gave a sensibility of $75 \%$ a specificity of $92 \%$ and an accurancy of $91.6 \%$.

The evaluation of the multiple parameters of NST, permits an easy and objective reading of the tracings independently of the presence of fetal movements. It facilitates the analysis of the tracings of dowbtful reactivity and unifies criteria of interpretation in order to compare studies betwen groups and institutions.

KEY WORDS: Nonstress testing, interpretation systems.

\section{Introducción}

Las pruebas sin estrés, se originaron hace más de 20 años, a raíz de las observaciones de Hammacher (1) y Kubli (2), en las que se relacionó la aparición de aceleraciones de la frecuencia cardíaca fetal (FCF) con la presencia de bienestar fetal. La prueba clínica como tal, fue divulgada hacia mediados de los años setenta, con base a los trabajos de Rochard (3) y Lee (4).

Pese a que el Test de no estrés (TNS) se ha convertido en un método universalmente aceptado de vigilancia fetal, un problema inherente a la prueba ha sido la falta de estándares para su interpretación.

Aunque suele considerarse «reactiva» una prueba que presente dos o más aceleraciones de la FCF de al menos 15 latidos por minuto y duración de 15 o más segundos, en un registro de 20 minutos, la valoración de este parámetro

\footnotetext{
* Médico Ginecobstetra. Centro Médico Sigma. Popayán.

** Médica Auxiliar. Centro Médico Sigma. Popayán.

*** Profesor Titular. Departamento de Estadística. Facultad de Ciencias de la Salud. Universidad del Cauca.
}

aislado, puede eventualmente conducir a un elevado número de pruebas «no reactivas» en fetos normales.

Varias revisiones clínicas, han considerado que los resultados de la interpretación de la prueba basados exclusivamente en la observación de aceleraciones reactivas, podrían mejorarse mediante la valoración de parámetros múltiples (5-6). Analizar los trazados de la FCF utilizando sistemas de puntuación de múltiples parámetros ha sido llamativo desde hace varias décadas. Hon en 1967 (7) y Schiffrin en 1972 (8) relacionaron los resultados de sistemas de puntuación de los trazados de la FCF durante el trabajo de parto, con la puntuación de Apgar. De igual manera, desde 1978 se ha pretendido valorar las pruebas de no estrés utilizando sistemas de calificación similares, que asignan valores matemáticos a los distintos parámetros del trazado (9-10).

En 1986, Bracero, Schulman y Baxi (11) publicaron un sistema de puntuación de los trazados de la FCF durante el trabajo de parto, derivado de las observaciones de Fischer, Hammacher y Krebs (12). El sistema tiene la ventaja de analizar el trazado como una onda sinusoidal independiente de los movimientos fetales y de la respuesta fetal a la contracción. 
Este método de valoración de los trazados de la FCF fue aplicado a la interpretación del TNS en el presente estudio. Los resultados obtenidos mediante este sistema, fueron comparados con la interpretación de la prueba que utiliza exclusivamente criterios de reactividad.

\section{Pacientes y métodos}

Entre octubre de 1990 y enero de 1992, se analizaron prospectivamente $142 \mathrm{TNS}$, realizados en 90 pacientes como parte integral de un programa de vigilancia fetal anteparto.

Cada prueba incluyó el registro de la FCF durante un período de 20 minutos y entre una y dos horas postingesta materna. Las pacientes fueron colocadas en posición semisentada y un observador corroboró permanentemente la actividad fetal. En todos los casos se utilizó un monitor Sonicaid-FM7L con una velocidad del papel de $3 \mathrm{cms}$ por minuto.

Las pruebas fueron evaluadas con base al sistema de puntuación descrito por Bracero y col. (11) y la conducta obstétrica fue tomada de acuerdo con los resultados de la puntuación.

\section{El sistema de puntuación}

El sistema consta de cinco variables, sobre las cuales se analiza la onda de la FCF, considerada simplemente como un trazado ondulatorio, independiente de la actividad fetal (tabla 1).

Tabla 1

SISTEMA DE PUNTUACION PARA LA FRECUENCIA CARDIACA FETAL* (1)

\begin{tabular}{|c|c|c|c|}
\hline Parámetro & $\mathbf{P} \quad \mathbf{u}$ & $\mathbf{n}$ & a j \\
\hline & 0 & 1 & 2 \\
\hline Frecuencia cardíaca basal & $\begin{array}{l}<100 \\
>180\end{array}$ & $\begin{array}{l}100-119 \\
161-180\end{array}$ & $120-160$ \\
\hline Frecuencia de las oscilaciones* (2) & $<3$ & $3-6$ & $>6$ \\
\hline Amplitud & $<5$ & $5-10$ & $>10$ \\
\hline Aceleraciones & 0 & $1-3$ & $>3$ \\
\hline Desaceleraciones* (3) & $>3$ & $1-3$ & 0 \\
\hline
\end{tabular}

*(1) Adaptado de Bracero, LA. Derivado de Fischer, Hammcher y Krebs *(2) Frecuencias mayores de 18 se les asigna una puntuación de 0.

*(3) A una desaceleración mayor de 2 minutos se le asignará una puntuación de 0.

1. Frecuencia cardíaca basal. La línea basal es observada en cada uno de los cuadros, determinando un promedio para todos ellos. En adelante el valor obtenido no debe cambiarse entre los cuadros, puesto que sobre él se van a calcular las demás variables. Frecuencias basales entre 120 y $160 \mathrm{lat} / \mathrm{min}$ se consideran normales y se les asigna una puntuación de dos. Frecuencias menores de 100 o mayores de 180 lat/min son patológicas y reciben una puntuación de cero. Los valores intermedios de la FCF que fluctúan entre 100 y 119 ó 161 y 180 lat/min se califican con un punto.

2 Frecuencia de las oscilaciones. El análisis de la frecuencia y amplitud de las oscilaciones se realiza de acuerdo con los parámetros delineados por Jagani y cols. (13) (figura 1). La frecuencia de las oscilaciones se calcula contando el número de veces que la onda atraviesa la línea basal hacia arriba o hacia abajo, en un período de un minuto.
Figura 1

CALCULO DE LA FRECUENCIA Y AMPLITUD DE LAS OSCILACIONES

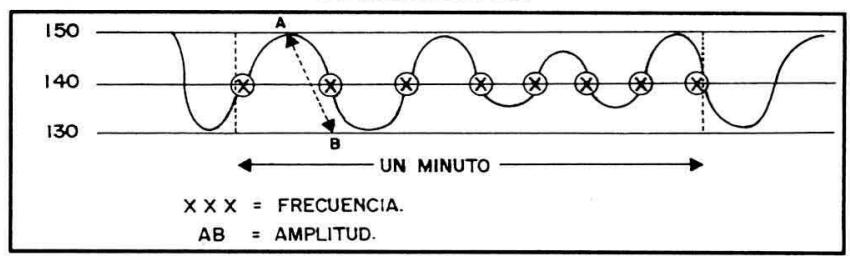

Ilustración de la forma en que se calcula la frecuencia y la amplitud de las oscilaciones. (Adaptado de Jagani y Col.).

Frecuencias por debajo de tres son anormales y se califican con cero. Frecuencias entre tres y seis se puntúan con uno y valores mayores de seis se consideran normales y se les asignan dos puntos. Oscilaciones mayores de 18 corresponden a ruido electrónico y se califican con cero. Los trazados sinusoidales reciben una puntuación de cero.

3. Amplitud de las oscilaciones. En un segmento de un minuto en el que no hayan aceleraciones ni desaceleraciones, se seleccionan los puntos de máxima y mínima oscilación en la onda de mayor amplitud. El número de latidos de la fluctuación, obtenido al restar el punto más bajo del más alto constituye la amplitud (figura 2). Valores mayores a diez latidos se consideran normales y se les asignan dos puntos. Amplitudes entre cinco y diez latidos se califican con un punto y menores de cinco latidos con cero.

Figura 2

ESTIMACION DE LA FRECUENCIA Y AMPLITUD EN UN TRAZADODE LA FCF

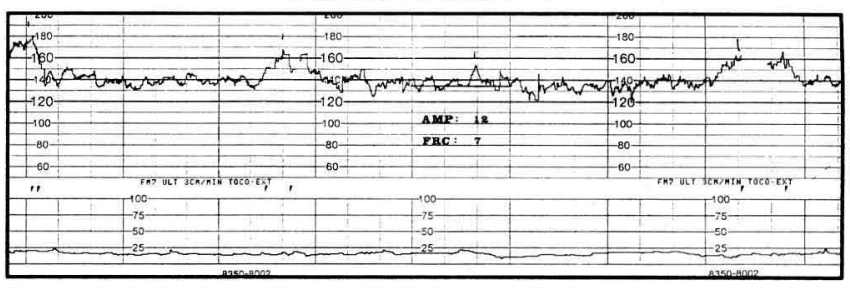

Estimación de la frecuencia y amplitud de las oscilaciones en un trazado de la frecuencia cardíaca fetal.

4. Aceleraciones. Corresponden a fluctuaciones de la FCF por encima de la línea basal de más de 15 lat/min y de más de 15 segundos de duración, contados desde el momento en que la onda se desprende de la basal hasta cuando regresa a ella. Si en el trazado de 20 minutos se observan más de tres aceleraciones se asignan dos puntos. La presencia de una a tres aceleraciones se califica con un punto y la ausencia de ellas con cero.

5. Desaceleraciones. A la inversa de las aceleraciones, corresponden a fluctuaciones de la FCF por debajo de la línea basal, de más de 15 lat/min y de más de 15 segundos de duración. La ausencia de desaceleraciones se califica con dos puntos. La presencia de una a tres desaceleraciones con un punto y más de tres desaceleraciones con cero. La presencia de una desaceleración de más de dos minutos se puntúa con cero.

La respuesta a los movimientos fetales o las modificaciones del trazado con las ocasionales contracciones de Braxton Hicks, no fueron tenidas en cuenta para la puntuación ni la conducta obstétrica.

Puntuaciones de siete o más fueron consideradas normales y relacionadas con bienestar fetal. Los puntajes de seis o 
menos se consideraron patológicos y el embarazo fue terminado, siempre y cuando existiera madurez pulmonar fetal.

El análisis de los resultados de la prueba se basó en estrechos criterios confirmatorios de la presencia o ausencia de bienestar fetal. Para la valoración de la prueba no fueron considerados calificadores posteriores al parto vaginal, tales como puntuación de Apgar, hospitalización en Unidad de Neonatología o compromiso neurológico fetal, por considerar que estos resultados son demasiado distantes de la prueba y están más relacionados con eventos propios del parto que el Test no está en capacidad de predecir.

Se consideraron como criterios indicativos de bienestar fetal: 1) Sobrevida fetal siete días después de la prueba. 2) Sobrevida fetal hasta el inicio del trabajo de parto cuandoéste ocurrió antes de cumplirse siete días de la prueba. 3) Nacimiento de un feto sano con Apgar igual o mayor de siete, cuando el embarazo terminó por cesárea electiva, en los siete días posteriores a la prueba.

Fueron considerados como criterios indicativos de pérdida del bienestar fetal: 1) Muerte fetal en los siete días posteriores a la prueba. 2) Muerte fetal antes del inicio del trabajo de parto, cuando éste ocurrió antes de cumplirse siete días de la prueba. 3) Feto deprimido con Apgar igual o menor de seis, cuando el embarazo terminó en cesárea electiva y la depresión fetal no fue debida a influencia anestésica o dificultad técnica en la extracción.

De igual manera se consideró como pérdida del bienestar fetal aquellos casos en que existía compromiso aparente de la salud fetal que amenazara potencialmente su vida, tales como retardo en el crecimiento fetal severo o insuficiencia placentaria.

\section{Resultados}

Tres pacientes y diez pruebas fueron excluidas del análisis por distintas razones: cinco pruebas correspondían a dos pacientes que recibían Terbutalina en el momento de la prueba. Tres test realizados en una paciente cuyo producto presentaba una disritmia cardíaca, con hallazgos ecocardiográficos sugestivos de cardiopatía congénita y dos pruebas efectuadas en dos pacientes que recibían Fenoterol y Fenobarbital, fueron igualmente excluidas.

Las 132 pruebas restantes, realizadas en 87 pacientes, constituyen el grupo de estudio. Los aspectos clínicos y epidemiológicos se resumen en la tabla 2 .

La edad materna promedio fue de 28 años y el $35 \%$ de las embarazadas eran primigestantes. Cerca de la mitad del grupo estaba constituido por embarazos de alto riesgo, hecho que probablemente incidió en un elevado índice de cesáreas (69\%).

La mayoría de las pruebas fueron realizadas alrededor de la semana 38 de gestación, pero el intervalo prueba-parto fue muy variable con un promedio de 12 días.

El peso promedio al nacer fue de 3.279 gr y el $50.5 \%$ de los recién nacidos fue de sexo masculino. Se registraron 12 casos de sufrimiento fetal (13.7\%) y el $8 \%$ de los recién nacidos presentó puntuaciones de Apgar al minuto bajas, iguales o menores a seis. Sin embargo a los 5 minutos sólo un recién nacido continuó deprimido. No se registró mortalidad perinatal en el grupo estudiado.

Alrededor del $20 \%$ de los casos presentó o bien líquido amniótico meconiado o calcificaciones macroscópicas de la placenta o circulantes de cordón.
Tabla 2

ASPECTOS CLINICOS DEL GRUPO ESTUDIADO

(PACIENTES: $\mathbf{N}^{\circ}=87$ - YNS: $\mathbf{N}^{\circ}=\mathbf{1 3 2}$ )

\begin{tabular}{|lrrcc|}
\hline Variable & n & \% & Promedio & DS \\
\hline Edad materna (Años) & 87 & & 28.0 & 5.60 \\
Edad gestacional (Semanas) & 132 & & 38.1 & 1.95 \\
Primigestantes & 31 & 35.6 & & \\
Embarazo alto riesgo & 40 & 45.9 & & \\
Nacimiento por cesárea & 60 & 68.9 & & \\
Productos de sexo masculino & 44 & 50.6 & & \\
Intervalo prueba-parto & 132 & & 12.2 & 12.01 \\
Peso fetal (Gramos) & 87 & & 3.279 & 498.9 \\
Sufrimiento fetal & 12 & 13.7 & & \\
Apgar bajo al minuto ( 6) & 7 & 8.0 & & \\
Apgar bajo al 5 ${ }^{\circ}$ minuto (6) & 1 & 1.1 & & \\
Líquido amniótico meconiado & 19 & 21.8 & & \\
Circulares de cordón & 18 & 20.6 & & \\
Calcificaciones placentarias & 18 & 20.6 & & \\
Macroscópicas & & & & \\
Mortalidad perinatal I & 0 & & & \\
\hline
\end{tabular}

En la tabla 3 se resumen los resultados del sistema de puntuación obtenidos en el grupo y los valores promedio para cada componente del sistema. La frecuencia cardíaca basal promedio fue de $139 \mathrm{lat} / \mathrm{min} \pm 7.7$. La frecuencia de las oscilaciones fue de 9.8 en un minuto y la amplitud de 14 latidos.

En 124 pruebas (94\%) se observaron aceleraciones de la FCF con un promedio de 5.2 aceleraciones por prueba. En 61 pruebas $(46 \%)$ se observaron desaceleraciones variables que ocurrieron aproximadamente con una frecuencia de 2,5 desaceleraciones por prueba. Se presentaron tres casos de bradicardia fetal prolongada.

Tabla 3

COMPONENTES INDIVIDUALES DEL TNS EN EL GRUPO ESTUDIADO

\begin{tabular}{|lrcc|}
\hline & n & Promedio & DS \\
\hline Frecuencia cardíaca basal & 132 & 139.3 & 7.7 \\
Frecuencia de las oscilaciones & 132 & 9.8 & 2.7 \\
Amplitud & 132 & 14.0 & 4.1 \\
Aceleraciones & 124 & 5.2 & 3.2 \\
Desaceleraciones variables & 61 & 2.5 & 2.0 \\
Bradicardia fetal prolongada & 3 & - & - \\
\hline
\end{tabular}

De las 132 pruebas analizadas, $128(97 \%)$ se consideraron reactivas con puntajes de siete o más. Cuatro pruebas (3\%) se calificaron como no reactivas con puntuaciones de seis o menos (Tabla 4). Estos 4 productos con pruebas no reactivas fueron evacuados por cesárea, bajo la hipótesis de pérdida del bienestar fetal y riesgo de muerte fetal. Al evaluar el estado fetal al nacer, se comprobó que dos de ellos (50\%) presentaban una patología acorde con el resultado de la prueba: uno de ellos (caso $\mathrm{N}^{\circ} 24$ ) nació deprimido y presentaba un cordón umbilical extremadamente corto. El otro (caso $\mathrm{N}^{\circ} 61$ ) padecía un retardo severo en el crecimiento fetal. Sin embargo, los otros dos recién nacidos (casos $\mathrm{N}^{\circ} 8$ y $\mathrm{N}^{\circ} 11$ ) en quienes el test fue anormal, fueron considerados como sanos. 
Tabla 4

COMPONENTES INDIVIDUALES DEL TNS Y ESTADO FETAL AL NACER DE LAS CUATRO (4) PRUEBAS CONSIDERADAS "NO REACTIVAS"

\begin{tabular}{|c|c|c|c|c|c|c|c|c|c|}
\hline Pte $\mathbf{N}^{\circ}$ & $\begin{array}{l}\text { Intervalo } \\
\text { Prueba-Parto }\end{array}$ & Basal & Frec Ampl. & Acel. & Desac. & Puntaje & Apgar & $\begin{array}{l}\text { Rectén } \\
\text { nacido }\end{array}$ & \\
\hline 8 & 1 & 155 & 5 & 8 & 0 & 0 & 06 & $10-10$ & Sano \\
\hline 11 & 0 & 162 & 5 & 28 & 2 & $1 \times 2^{1}$ & 05 & $10-10$ & Sano \\
\hline 24 & 0 & 140 & 9 & 5 & 1 & $2 / 1 \times 5^{1}$ & 06 & $5-9$ & $\begin{array}{l}\text { Deprimido. } \\
\text { Condónoanto. }\end{array}$ \\
\hline 61 & 0 & 158 & 8 & 10 & 0 & 7 & 05 & $9-10$ & RCIUsevero. \\
\hline
\end{tabular}

Por otra parte cuatro recién nacidos cumplieron con los requisitos previamente establecidos como indicativos de pérdida del bienestar fetal. De igual manera estos cuatro productos nacieron por cesárea. $\mathrm{Al}$ analizar sus trazados (tabla 5), dos recién nacidos afectados (50\%) habían presentado una prueba anormal, acorde con su situación clínica. Uno de ellos (caso $\mathrm{N}^{\circ} 24$ ) nació deprimido con un cordón extremadamente corto; el otro (caso $\mathrm{N}^{\circ} 61$ ) presentaba un retardo en el crecimiento fetal intrauterino, severo. Sin embargo, la prueba anteparto resultó normal en dos fetos $(50 \%)$ aparentemente afectados. Uno de ellos (caso $\mathrm{N}^{\circ} 46$ ) presentó sufrimiento fetal en el preparto y nació por cesárea, deprimido y meconiado. El otro (caso $\mathrm{N}^{\circ} 67$ ), nació igualmente deprimido y presentaba una insuficiencia placentaria.

Tabla 5

COMPONENTES INDIVIDUALES DEL TNS Y ESTADO FETAL AL NACER EN LOS CUATRO PRODUCTOS EN QUIENES SE CONFIRMO PERDIDA DEL BIENESTAR FETAL

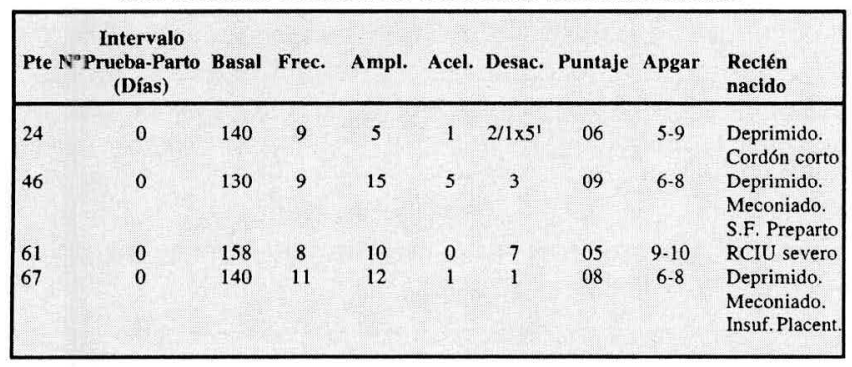

La primera parte del análisis se orientó a determinar la exactitud del sistema de calificación de parámetros múltiples del TNS. Se encontró que una puntuación anormal de seis o menos, acierta en el $50 \%$ de los casos con la presencia de un feto comprometido. Sin embargo la mitad de los fetos con una prueba anormal, serán sanos. Por otra parte, sólo la mitad de los fetos afectados, tendrán una prueba anormal; la otra mitad mostrarán pruebas normales.

El análisis estadístico demuestra que el sistema de calificación de parámetros múltiples del TNS tiene una sensibilidad del 50\%; una especificidad del $98.4 \%$; un valor predictivo positivo del $50 \%$ y un valor predictivo negativo del $98.4 \%$. La exactitud del sistema es del 96.9\% (tabla 6).

En una segunda parte del análisis se buscó la exactitud de la prueba cuando fue interpretada utilizando únicamente criterios de reactividad. Se consideró como prueba reactiva aquella que presentaba al menos dos períodos de aceleración de la FCF, de más de $15 \mathrm{lat} / \mathrm{min}$ y más de 15 segundos de duración en el trazado de 20 minutos. No se consideró la respuesta fetal a los movimientos y el resultado de este análisis no fue tenido en cuenta para la definición de la conducta obstétrica.

Tabla 6

ANALISIS ESTADISTICO DEL SISTEMA DE PUNTUACION DE PARAMETROS MULTIPLES

Patología confirmada

-pérdida del bienestar fetal-

\begin{tabular}{|c|c|c|c|}
\hline$\sum_{\underline{I I}}^{0}$ & & SI & NO \\
\hline 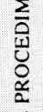 & $\begin{array}{c}+ \\
\text { TNS } \\
\text { No reactivo }\end{array}$ & $\begin{array}{c}\text { Verdaderos positivos } \\
2\end{array}$ & $\begin{array}{c}\text { Falsos positivos } \\
2\end{array}$ \\
\hline 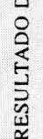 & $\begin{array}{c}\text { TNS } \\
\text { Reactivo }\end{array}$ & $\begin{array}{c}\text { Falsos negativos } \\
2\end{array}$ & $\begin{array}{c}\text { Verdaderos negativos } \\
126\end{array}$ \\
\hline
\end{tabular}

Sensibilidad: $50 \%$ Especificidad: $98.4 \%$ Valor predictivo positivo: $50 \%$ Valor predictivo negativo: $98.4 \%$. Exactitud: $96.9 \%$.

De las 132 pruebas valoradas con este sistema, 119 resultaron reactivas $(90.1 \%$ ) y 13 no reactivas $(9.9 \%)$. De las 119 pruebas reáctivas, $118(99.1 \%)$ coincidieron con fetos normales y sólo una $(0.9 \%)$ resultó normal en un producto afectado. Sin embargo como se ilustra en la tabla 7, de las 13 pruebas consideradas no reactivas, sólo tres (23\%) correspondían a productos verdaderamente afectados y diez (77\%) a fetos sanos (falsos positivos).

El análisis estadístico demuestra que si la prueba se interpreta únicamente por criterios de reactividad, se mejora la sensibilidad (75\%) al tiempo que se pierde en especificidad $(93 \%)$. Se tiene entonces un excelente valor predictivo negativo del $99.1 \%$, pero un pobre valor predictivo positivo del $23 \%$. La exactitud de la prueba analizada de esta manera, es del 91.6\% (tabla 7).

Tabla 7

\section{ANALISIS ESTADISTICO DE LA INTERPRETACION DE LOS MISMOS TRAZADOS UTILIZANDO CRITERIOS DE REACTIVIDAD}

(CUANTIFICACION DE ACELERACIONES)

Patología confirmada -pérdida del bienestar fetal-

\begin{tabular}{|c|c|c|c|}
\hline \multirow{3}{*}{ 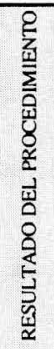 } & \multicolumn{2}{|r|}{ SI } & NO \\
\hline & $\begin{array}{c}+ \\
\text { TNS } \\
\text { No reactivo }\end{array}$ & $\begin{array}{c}\text { Verdaderos positivos } \\
3\end{array}$ & $\begin{array}{c}\text { Falsos positivos } \\
10\end{array}$ \\
\hline & $\begin{array}{c}\text { TNS } \\
\text { Reactivo }\end{array}$ & $\begin{array}{c}\text { Falsos negativos } \\
1\end{array}$ & $\begin{array}{l}\text { Verdaderos negativos } \\
118\end{array}$ \\
\hline
\end{tabular}

Sensibilidad: $75 \%$ Especificidad: $92 \%$ Valor predictivo positivo: $23 \%$ Valor predictivo negativo: $99 \%$. Exactitud: $91.6 \%$.

Al comparar los dos sistemas de análisis, (tabla 8), se observa que si la interpretación del TNS se hace utilizando criterios de reactividad se aumentan las pruebas verdaderas positivas y se disminuyen los resultados falsos negativos 
pero a expensas de un considerable incremento en los resultados falsos positivos que llevarán a intervenciones innecesarias.

\section{Tabla 8}

COMPARACION ENTRE LOS DOS SISTEMAS DE ANALISIS DEL TNS

\begin{tabular}{|lcccccc|}
\hline Sistema de análisis & Sens. & Esp. & Vpp. & Vpn & $\begin{array}{c}\text { Falsos } \\
\text { Positivos }\end{array}$ & Exact. \\
Parámetros múltiples & 50 & 98.4 & 50 & 98.4 & 1.5 & 96.9 \\
Reactividad & 75 & 92.1 & 23 & 99.1 & 7.5 & 91.6 \\
\hline
\end{tabular}

El sistema de calificación de parámetros múltiples, aunque no tiene una sensibilidad mayor del $50 \%$, tiene en cambio una especificidad y un valor predictivo negativo muy elevados (98.4\%). La exactitud de este sistema es de $96.9 \%$ comparado con el $91.6 \%$ si se utilizan criterios de reactividad.

\section{Discusión}

La prueba sin estrés se ha constituido en un método universalmente aceptado de vigilancia fetal, por ser un procedimiento fácil de realizar, de interpretación rápida y carente de contraindicaciones. A pesar de que la prueba tiene una alta especificidad, su baja sensibilidad y su escaso valor predictivo positivo han sido decepcionantes (14). Aunque en la práctica clínica se espera que un test de detección de daño fetal tenga una alta sensibilidad, es decir que casi todos los fetos afectados presenten un resultado anormal (15), la exactitud de una prueba varía de acuerdo a como se la interprete y en función de la condición que se pretenda diagnosticar.

Múltiples factores pueden estar involucrados en la correlación de la capacidad predictiva del TNS. Por una parte, cabe recordar que la frecuencia con la cual una observación predice a la siguiente, depende no solamente de la prueba de valoración que se haya empleado, sino también de la frecuencia del transtorno en la población estudiada (15). No es lo mismo valorar una prueba de detección de daño fetal en una población de bajo riesgo donde la mortalidad fetal será muy baja, que en un grupo de pacientes de alto riesgo portadoras de patologías asociadas a un alto índice de muerte fetal .

Por otro lado, en la valoración de toda prueba clínica debe tenerse en cuenta, que a medida que se estrechen los parámetros de normalidad, aumentará el número de resultados falsos positivos. Así, como lo demostramos en el presente estudio, si se consideran como «normales» únicamente las pruebas que presenten aceleraciones reactivas de la FCF, se detectará la mayor parte de los fetos afectados, pero muchas de las pruebas anormales corresponderán a fetos sanos.

De igual manera, el clínico debe conocer las limitaciones de todo procedimiento diagnóstico. El TNS es incapaz de simular las condiciones del parto, por lo que la capacidad predictiva de la prueba no puede valorarse en función a situaciones clínicas posteriores a tal evento como puntuación de APGAR o daño neurológico fetal. Es muy posible que la mortalidad y morbilidad fetal provenientes de otras condiciones no predicibles con la prueba hayan contribuido a distorsionar su veracidad.

Por consiguiente, al valorar la exactitud de una prueba preparto de bienestar fetal deben establecerse parámetros precisos de verificación de esa condición. Confirmar el resultado de las pruebas anormales, puede ser en la práctica clínica más sencillo que el de las pruebas normales. Si ante una prueba anormal el feto se evacúa por cesárea es factible confirmar si su estado al nacer concuerda con el resultado de la prueba. Confirmar si una prueba normal se correlaciona verdaderamente con un feto sano suele ser más complejo. Puesto que no es posible la verificación inmediata de la condición fetal debe recurrirse a parámetros indirectos de bienestar como sobrevida un tiempo después de la prueba, ausencia de patología fetal o placentaria aparente etc.

A pesar de las anteriores consideraciones, el problema crucial de las pruebas sin estrés ha sido la carencia de estándares para su interpretación (16) y la falta de un consenso uniforme sobre la nomenclatura o el lenguaje que debe usarse para describir los trazos de la FCF (11). Varios estudios han asegurado que los inconvenientes en la interpretación de la prueba basados exclusivamente en la observación de aceleraciones reactivas, podrían mejorarse mediante la valoración de parámetros múltiples $(6,16)$. Hon (7) Shiffrin (8) y Krebs (12) utilizaron sistemas de puntuación de los trazados de la FCF durante el trabajo de parto en los minutos previos a la expulsión y buscaron su correlación con índices de Apgar y bienestar fetal. Encontraron que globalmente los sistemas de puntuación son un buen predictor del desenlace fetal, lo cual es de esperar, ya que los sistemas tienen en cuenta múltiples parámetros de la FCF.

La pérdida de aceleraciones reactivas, así como la alteración aislada de cualquiera de los parámetros de la FCF, no son necesariamente indicativos de pérdida del bienestar fetal. Múltiples factores no patológicos, pueden modificar aisladamente cualquiera de las variables de un trazado de la FCF.

Así, en ausencia de otras características anormales, la taquicardia o la bradicardia fetal no se asocian a acidosis (12, 17-18). La taquicardia fetal puede ser debida a movimiento fetal, uso o consumo materno de simpaticomiméticos o parasimpaticolíticos, fiebre o ansiedad materna, corioamnionitis, anemia fetal, hipertiroidismo materno o falla cardíaca fetal.

La bradicardia fetal de 100 a $120 \mathrm{lat} / \mathrm{min}$ no se asocia a acidosis cuando la variabilidad es normal (19). La bradicardia fetal puede asociarse a postmadurez y posiciones occipitotransversa y occipito-posterior (19). La bradicardia fetal severa de menos de 100 lat/min se asocia con mayor frecuencia a bloqueos cardíacos congénitos, enfermedades maternas del colágeno, algunas anomalías cardíacas fetales e hipotermia materna (20).

Sin embargo, si estas mismas alteraciones de la línea basal bien sea taquicardia o bradicardia, se asocian a otras anormalidades del trazado, aumentan la sospecha de hipoxia fetal $(17,21)$.

Una variabilidad normal predice un buen resultado para el recién nacido (22), un funcionamiento neurológico normal (23) y una puntuación de Apgar normal (8). Aunque su pérdida progresiva precede a la muerte fetal (24), la variabilidad puede alterarse por circunstancias distintas al daño fetal.

Una serie de factores no asociados a la hipoxia pueden disminuir la variabilidad. Los hipnóticos y narcóticos sedantes como meperidina, morfina, metadona, barbitúricos, 
diazepam y alfaprodina, disminuyen la variabilidad (25-26), al igual que los anestésicos generales o locales y los agentes parasimpaticomiméticos (27).

La variabilidad disminuye con la prematurez. Las anormalidades del sistema nervioso central y el cambio del estado fetal de la alerta al sueño disminuyen en forma significativa la variabilidad (28).

La disminución aislada de la variabilidad sin la coexistencia de patrones de desaceleración, implica que no hay riesgo de acidosis o que éste es mínimo. En cambio, la disminución de la variabilidad combinada con desaceleraciones tardías o variables, identifica a los fetos con riesgo de acidosis a hipoxia (29-30).

El aumento de la variabilidad es la respuesta inicial a la hipoxia (31) y una variabilidad notablemente aumentada refleja una hipoxia temprana y posible acidosis (32). La variabilidad aumenta también con la postmadurez y la alerta fetal.

Las desaceleraciones por sí solas no indican daño fetal. No hay riesgo de acidosis o puntuaciones de Apgar bajas al minuto, mientras que la relación desaceleración-contracción en el trabajo de parto no exceda el $30 \%$ en desaceleraciones variables o el $20 \%$ en desaceleraciones tardías (33).

Las desaceleraciones tardías pueden presentarse con el uso de anestesia conductiva o la administración inadecuada de ocitocina (34). Sin embargo, cuando las desaceleraciones tardías se acompañan de ausencia de aceleraciones, disminución de la variabilidad o taquicardia, se asocian a acidosis fetal y puntuaciones de Apgar bajas hasta en el 65\% de los casos $(18,35)$.

El significado clínico de las desaceleraciones variables ha sido objeto de discusión. Aunque su incidencia en algunos estudios fluctúa entre 5 y $20 \%$ y se ha relacionado con disminución del volumen de líquido amniótico y compresión del cordón (36), otros sostienen una opinión distinta y afirman que las desaceleraciones variables no son signos de mal estado fetal y podrían presentarse hasta en el $50 \%$ de las pacientes y al menos en la mitad de los casos carecerían de significación clínica (37). En el presente estudio observamos una elevada frecuencia de desaceleraciones variables, presentes en el $46 \%$ de los trazados sin aparente repercusión clínica en la mayoría de ellos.

Los patrones sinusoidales, definidos como un trazado ondulante, con amplitud de por lo menos $10 \mathrm{lat} / \mathrm{min}$, con dos a cinco ciclos por minuto y duración mínima de 2 a 10 minutos (38-39), han sido relacionados con anemia fetal secundaria a insoinmunización $R h$ (40-41) y vinculados a una elevada mortalidad fetal $(3,40,42)$. Sin embargo el patrón sinusoidal se ha visto en ocasiones asociado a transfusiones uterinas (4344) y administración de alfaprodina (45).

La bradicardia fetal prolongada se ha relacionado con hipoxia secundaria a anomalías anatómicas del cordón, nudos de cordón, disminución de la gelatina de Warthon, compresión del cordón y oligoamnios en embarazos prolongados (46-48). Puede ocurrir sin embargo con la exploración vaginal el bloqueo paracervical, la manipulación fetal y la hipotensión materna (34).

De las consideraciones hasta aquí expuestas, parece deducirse que la alteración aislada de un sólo parámetro de la FCF no es un indicador de pérdida del Bienestar Fetal, como si puede serlo la alteración de varios de ellos.
Aunque la respuesta fetal a la hipoxia es mediada por mecanismos complejos, sigue más o menos un patrón predecible. La compresión inicial del cordón que origina obstrucción de la vena umbilical, reduce el retorno venoso al corazón fetal originando una caída en la presión sanguínea (49). Los baroreceptores del arco aórtico responden a esta señal produciendo una taquicardia transitoria compensadora (50). La situación clínica puede resolverse, continuar igual o agravarse. La disminución persistente del retorno venoso generará hipoxemia fetal que conducirá a cambios adaptativos compensadores. La respuesta inicial del feto sano a la disminución de la $\mathrm{PO}_{2}$ es una redistribución del gasto cardíaco a sus órganos vitales mediante vasoconstricción periférica mediada por quimioreceptores periféricos y secreción de catecolaminas. El reflejo vagal mediado por quimioreceptores se manifestará como desaceleraciones tardías de la FCF (50-51). La estimulación adrenérgica persistente en el feto, a diferencia de lo que sucede en el adulto llegará a ocasionar bradicardia (11). Si la compresión del cordón se agrava hasta producir oclusión completa, aparecerá hipertensión fetal y estimulación de los baroreceptores que ocasionará bradicardia (52).

Mientras exista hipoxemia sin hipoxia tisular la variabilidad estará conservada. Cuando la caída en la $\mathrm{PO}_{2}$ sobrepasa la reserva de oxígeno fetal, la hipoxia tisular resultante dará origen a un cambio del metabolismo aeróbico a anaeróbico con la consiguiente acidosis láctica y acidemia. Estos fenómenos originarán la pérdida de la variabilidad como manifestación de una insuficiente oxigenación cerebral y miocardia, así como depresión miocárdica hipóxica. (50-51).

Observaciones clínicas en fetos humanos de alto riesgo apoyan la aseveración de que la ausencia o disminución de las aceleraciones puede constituir el marcador temprano más sensible de hipoxia dentro del útero (53).

En la cascada teórica de acontecimientos secundarios a la descompensación fetal, la disminución de la amplitud y frecuencia de las aceleraciones es probablemente el primer acontecimiento. Posteriormente aparecerá disminución en la incidencia de los movimientos fetales y más tardíamente desacoplamiento entre aceleraciones y movimientos. Un compromiso más severo del estado fetal originará la pérdida de aceleraciones y movimientos y finalmente la aparición de desaceleraciones espontáneas (54).

Paul y col, evaluaron los cambios de la variabilidad en productos con desaceleraciones en sus trazados de FCF y correlacionaron sus observaciones con los valores de ph capilar. Observaron que aún con la presencia de desaceleraciones, los fetos que conservaban la variabilidad tenían grados menores de audosis que aquellos que la habían perdido (55).

Clark y col, revisaron 100 trabajos de parto consecutivos, en los que se estableció el diagnóstico de sufrimiento fetal con base en trazados anormales de la FCF. Todos los fetos fueron sometidos a tomas de muestra de sangre del cuero cabelludo para determinación de pH. Aquellos fetos que aceleraban la frecuencia cardíaca en respuesta a la estimulación del cuerocabelludo producida por el pinzamiento o la punción, tuvieron valores de $\mathrm{pH}$ de 7.20 o más. Ninguno de los fetos cuyo $\mathrm{pH}$ resultó inferior a 7.20 respondió a este estímulo (56).

Las consideraciones hasta aquí expuestas fundamentan el concepto de que la afección de múltiples variables en un 
trazado de la FCF conlleva un mayor riesgo y un peor pronóstico para el feto que la alteración aislada de una de ellas mientras las demás variables permanezcan normales.

$\mathrm{Al}$ suponer una relación directa y aditiva entre el número de variables afectadas y el daño fetal, tenemos la sustentación teórica para afirmar que los sistemas de calificación de parámetros múltiples pueden constituirse en un excelente método de valoración de los trazados de la FCF.

El sistema analizado en el presente estudio, permite establecer un modelo práctico, de fácil lectura e interpretación objetiva del TNS, que unifica criterios y facilita la comparación de estudios entre grupos e instituciones.

Debe tenerse en cuenta sin embargo, que a pesar de que una prueba reactiva con un puntaje de siete o más corresponde en el $98.4 \%$ de los casos a fetos sanos, una prueba no reactiva calificada con seis o menos puntos, corresponderá en el 50\% de los casos a fetos sanos y sólo detectará el 50\% de los fetos afectados.

Un feto afectado tendrá el $50 \%$ de probabilidades de ser detectado si se utiliza un sistema de calificación de parámetros múltiples y el $75 \%$ de probabilidad si se utilizan criterios de reactividad. Sin embargo, si en el análisis de los trazados se utilizan únicamente criterios de reactividad, una prueba reactiva corresponderá en el $99 \%$ de los casos a fetos sanos, pero la prueba no reactiva se correlacionará solamente en el $23 \%$ de los casos con un feto verdaderamente afectado.

Los dos sistemas de interpretación de la prueba aquí analizados deben de considerarse complementarios. El sistema de calificación de parámetros múltiples permitirá, no obstante, analizar con mayor facilidad ciertos trazados difíciles de interpretar cuando se utiliza únicamente un sistema convencional de reactividad.

El TNS continúa siendo un excelente método para la valoración del bienestar fetal y constituye indudablemente un predictor seguro de salud fetal, pese a que posee una escasa capacidad predictiva sobre los eventos propios del parto. Sea cual sea el método que se utilice en la interpretación de la prueba, ésta solo permitirá seleccionar productos con mayor riesgo que deben derivarse a pruebas adicionales de bienestar fetal.

\section{BIBLIOGRAFIA}

1. Hammacher R. The clinical significance of cardiotocography. In Huntingford, P., Hunter, M., Saling, E (Eds): Perinatal Medicine. New York, Academic Press, 1970; 80-93.

2. Kubli FW., Kaeser O., Hinselmann M. Diagnostic management of chronic placental insufficiency. In Pericle, A., Finzi, C (Eds): The foeto-placental unit. Amsterdam, Excerpta Medica Foundation 1969; 323.

3. Rochard F., Schifrin BS., Goupil F et al. Nonstressed fetal heart rate monitoring in the antepartum period Am. J. Obstet. Gynecol. 1976; 126: 699 .

4. Lee CY., Diloreto DC., Logrand B. Fetal activity acceleration determination for the evaluation of fetal reserve. Obstet. Gynecol. 1976; 48: 19 .

5. Devoe LD., Mckenzie J., Searle NS et al. Clinical-sequelae of the extended nonstress test. Am. J. Obstet. Gynecol. 1985; 151: 1074.

6. Devoe LD., Yanowitch G., Azor H. The aplication of multiple parameter scoring to antepartum fetal heart rate testing J. Reprod. Med. 1981; 26: 250.

7. Hon EH. Detection of fetal distress. In: Wood, C, ed. Fifth world congress of gynecology and obstetrics. Sydney Australia: Butterworths 1967; 58:

8. Schiffrin BS., Dame L. Fetal heart rate patterns. JAMA 1972; 219: 1322.

9. Pearson JF., Weaver J. A six point scoring system for antenatal cardiofocographs. British Journal of Obstetrics and Gynaecology 1978; 85: 321.

10. Lyons ER., Bylsma-Howell M., Shamsi S., Towell ME. A scoring system for nonstressed antepartum fetal heart rate monitoring. Am. J. Obstet. Gynecol. 1979; 133: 242.

11. Bracero LA., Shulman H., Baxi LV. Características de la frecuencia cardíaca fetal que apoyan el diagnóstico de Bienestar Fetal. Clínicas Obstétricas y Ginecológicas 1986; 1: 3.

12. Krebs HB., Petres RE., Dunn LJ et al. Intrapartum fetal heart rate monitoring II-Multifactorial analysis of intrapartum fetal heart rate tracings. Am. J. Obstet. Gynecol. 1979; 133: 773.

13. Jagani N., Schulman H., Fleischer A et al. The value of quantitative analysis of fetal heart rate tracings. Perinatol-Neonatol 1983; 7: 55.

14. Devoe LD. Prueba sin estrés. Clínicas de Ginecología y Obstetricia. Temas actuales 1990; 1: 107.

15. Lame Jay D., Reiss, Rosemary. ¿Cuándo debe interrumpirse el parto para practicar un alumbramiento mediante cesárea? Clínicas Obstétricas y Ginecológicas 1985; 4: 937.
16. Devoe LD., Castillo RA., Sherline DM. The nonstress test as a diagnostic test. A critical reappraisal. Am. J. Obstet. Gynecol. 1985; 152: 1047.

17. Tejani N., Mann LI., Brakthavathsalan A., Weiss RR. Correlation of fetal heart rateuterine contraction patterns with scalp blood ph. Obstet. Gynecol. 1975; 46: 392.

18. Kubli FW., Hon EK., Khazin AF., Jakemura H. Observation on heart rate and $\mathrm{ph}$ in the human fetus, during labor. Am. J. Obstet. Gynecol. 1969; 104: 1190.

19. Young BK., Katz M., Klein SA., Silverman F. Fetal blood on tissue ph with moderate bradycardia. Am. J. Obstet. Gynecol. 1979; 135: 45.

20. Pritchard JA., Macdonald PC., Gant NF. Williams obstetrics 17 th ed, Norwalk Appleton-Century Crofts 1984; 286.

21. Beard RW., Filshle GM., Khight CA. The significance of the changes in the continuous fetal heart rate in the first stage of labour. J. Obstet. Gynaecol. Br. Conmonw 1971; 78: 865.

22. Yeh SY., Forsythe A., Hon EH. Quantification of fetal heart heat to heat interval differences. Obstet. Gynecol. 1973; 41: 355.

23. Benson RC., Shuberk F., Deutschberger J., Weiss W., Berendes H. FHR as predictor of fetal distress: a report from the colaborative project. Obstet. Gynecol. 1968; 32: 259.

24. Centrulo CL., Schifrin BS. FHR patterns proceding death in utero. Obstet. Gynecol. 1976; 48: 521.

25. Petrie RH., Yeh S., Murata Y et al. The effect of drugs on fetal heart rate variability. Am. J. Obstet. Gynecol. 1978; 130: 294.

26. Yeh SY., Paul RH., Cordero LA. Study of diazepam during labor. Obstet. Gynecol. 1974; 43: 363.

27. Schifferli PY., Caldeyro, Barcia R. Effect of atropine and beta adrenergic frugs on the heart rate of the human fetus. In Boreus, L.O. ed. Fetal pharmacology. New York. Raven Press: 1972; 259.

28. Martin CB. Physiology and clinical use of fetal heart rate variability. Clin perinatol 1982; 9: 339.

29. Zanini B., Paul RH., Huey JR. Intrapartum fetal heart rate correlation with scalp ph in the preterm fetus. Am. J. Obstet. Gynecol. 1980; 136: 43.

30. Paul RH., Suidman AR., Yeh SY., Schiffrin BS., Hon EH. Clinical fetal monitoring III. The evaluation and significance of intrapartum baseline fetal heart rate variability. Am. J. Obstet. Gynecol. 1975; 123: 206.

31. Hammacher K., Huter KA., Bokelman J., Werner PH. Foetal heart frecuency and condition of the foetus and newhorn. Ginecologia 1968; 166: 349. 
32. Druzin ML., Ikenouse T., Murata Y et al. A possible mechanism for the increase in fetal heart rate variability folowing hipoxemia. Presented of the Society for Gynecologic Investigation. San Diego California, 1979.

33. Lin CC., Schulman H., Saldana LR. Deceleration/contraction ratios as an index of fethal halth during labor. Obstet. Gynecol. 1978; 51: 666.

34. Quirk G., Miller F. Características de los registros de la FCF que ponen en peligro al feto. Clin. Obstet. y Gin. 1986; 1: 15.

35. Krebs HB., Petres RE., Dunn LJ., Jordan HV., Segreti A. Intrapartum fetal heart rate monitoring I. Clasification and prognosis of fetal heart rate patterns. Am. J. Obstet. Gynecol. 1979; 133: 762.

36. Phelan JP., Lewis PE. Fetal heart rate deceleration during a nonstress test. Obstetrics and Gynecology 1981; 57: 228.

37. Meiss PJ., Ureda JR., Swan M et al. Variable deceleration are not a sign of fetal compromise. American Journal of Obstetrics and Gynecology 1986; 154: 586.

38. Leveno KJ., Quirk JG., Cunningham FG et al. Prolonged pregnancy I. Observations conserning the causes of fetal distress. Am. J. Obstet. Gynecol. 1984; 150: 465.

39. Johnson TRB., Compton AA., Rotmensch J., Work BA., Johnson JW. Significance of the sinusoidal fetal heart rate pattern. Am. J. Obstet. Gynecol. 1981; 139: 446.

40 . Kubli F., Ruttgers H. Haller. Die antepartale fetale herzfrequenz. II. Verhalten von Grundfreguenz fluctuation und Dezerationen hei antepartalem Fruchttod. Z, Gehurtshilfe Perinatol 1972; 176: 309.

41. Hatjis CG., Mennuti MT., Sack LM., Schwarz RZ. Resolution of a sinusoidal fetal heart pattern following intrauterine transfusion. Am. J. Obstet. Gynecol. 1978; 132: 109.

42. Manseau P., Vaquier J., Chavinie J. Lerythme cardiaque foetal «sinusoidal» J. Gynecol. Obstet. 1972; 1: 343.

43. Lowe TW., Leveno KJ., Quirk JG., Santos Ramon R., Williams ML. Sinusoidal fetal heart rate pattern after intrauterine transfusion. Obstet. Gynecol. 1984; 64: 215.
44. Mueller-Heubach E., Caritis SN., Edelstone DI. Sinusoidal fetal heart rate pattern following intrauterine fetal transfusion. Obstet. Gynecol. 1978; $52: 43$.

45. Gray JH., Dumore DW., Luther ER. Sinusoidal fetal heart rate pattern associated with alphaprodine administration. Obstet. Gynecol. 1978; 52: 678 .

46. Buehm FH. Prolonged end stage fetal heart rate deceleration. Obstet Gynecol. 1975; 45: 579.

47. Herbert GM., Boehm FH. Prolonged end stage fetal heart rate deceleration: a reanalysis. Obstet. Gynecol. 1981; 57: 589.

48. Leveno KJ., Quirk JG., Cunningham FG et al. Prolonged pregnancy. I. Observations concerning the causes of fetal distress. Am. J. Obstet. Gynecol. 1984; 150: 465 .

49. James LS., Yeh MN., Morishima $\mathrm{HO}$ et al. Umbilical vein occlusion and trasient acceleration of the fetal heart rate. Am. J. Obstet. Gynecol. 1976; 126: 276.

50. Freman RK., Garite TJ. Fetal heart rate monitoring Baltimore: Williams \& Wilkins 1981; 7: 63.

51. Harris JL., Krueger TR., Parer JT. Mechanisms of late decelerations of the fetal heart rate during hipoxia. Am. J. Obstet. Gynecol. 1982; 144: 491.

52. Reece EA., Antoine C., Montgomery J. El feto como juez final de la presencia de estrés/sufrimiento intrauterino. Clin. Obst. y Gin. 1986 1: 47.

53. Vintzileos AM., Camphell WA., Inggardia CJ et al. The fetal biophysical profile and its predictive value. Obstet. Gynecol. 1983; 62: 271 .

54. Devoe LD. Prueba sin estrés. Clínicas de ginecología y obstetricia. Temas actuales 1990; 1: 107.

55. Paul RH., Suidman AK., Yeh SY., Schifrin BS., Hon EH. Clinical fetal monitoring III. The evaluation and significance of intrapartum baseline fetal heart rate variability. Am. J. Obstet. Gynecol. 1975; 123: 206.

56. Clark S., Gimovsky ML., Miller FC. The scalp blood sampling. Am. J. Obstet. Gynecol. 1984; 148: 272. 